\title{
Perspectives Perspectives
}

This section features: (1) reactions of readers to articles and reviews published in the Journal and the replies of authors to whom the comments are addressed (if forthcoming) and (2) viewpoints and opinions expressed in the form of a report, commentary, or interview on issues or topics of current interest.
Cette section sera consacrée à deux types d'articles:

1. La réaction des lecteurs aux articles parus dans la revue et la réponse de leurs auteurs, sill y a lieu.

2. Les points de vue et les opinions, présentés sous forme de comptes rendus, de commentaires, de chroniques ou d'entrevues, sur des sujets d'actualité ou d'intérêt général.

\section{LA PROBLEMATIQUE DE L'INTEGRATION DES ECOLIERS HAITIENS AU MILIEU SCOLAIRE QUEBECOIS}

\section{Jean Kerlegrand}

L'immigration haïtienne est tout à fait récente au Québec. Elle date de la dernière décennie. Avant cette époque, les quelques Haïtiens qui y arrivaient ne le faisaient que dans l'unique but de poursuivre des études universitaires, pour ensuite retourner chez eux. A la fin des années soixante et plus particulièrement lors de l'exposition universelle, "La Terre des Hommes," certains intellectuels et professionnels en ont profité pour échapper à la répression politique qui sévissait en Haïti. De plus, cette époque était aussi favorable à l'importation de cerveaux puisque, le Québec en pleine mutation sociale (la Révolution tranquille) avait besoin de techniciens, de spécialistes et de main-d'oeuvre pour son développement social, culturel et économique.

Cet exode Haïtien prit de l'ampleur et s'intensifia dans les années soixante-dix si bien qu'on assista à une prolifération d'hommes, de femmes, à la fois sans métiers et sous-scolarisés (certains d'entre eux ne savaient ni lire, ni écrire), à la recherche d'emploi ou de vie meilleure. Le chômage, la misère faisaient de leur pays (Haïti) une géhenne dans laquelle croupissaient la grande majorité des familles haïtiennes. On connaît la suite: les "boat people" et les "réfugiés de l'air" du début des 
années 80-les "réfugiés économiques"-comme certains se plaisent à les appeler et à les identifier.

Une fois installés au Québec, il fallait s'attendre à faire face aux réalités du pays, entre autres le contexte social et les conditions de vie difficiles. Effectivement, la communauté haïtienne du Québec souffre actuellement de multiples problèmes et connaît tous les maux évidents à une immigration de cette nature. Il y a un choc, dirait-on, le choc culturel agrémenté d'amères illusions, de faux espoirs et de nostalgie.

L'un des problèmes les plus frappants est celui de l'adaptation ou de l'intégration des élèves haïtiens au milieu scolaire québécois.

\section{Comment Comprendre le Problème?}

Répondre à la question revient à identifier les principales causes et les différentes situations auxquelles se trouvent confrontés les élèves haïtiens. Ces situations sont de nature complexe et variée.

Les enfants immigrants ont en général de la difficulté à s'intégrer ou à s'adapter au milieu scolaire québécois. Dans un article publié le 3 janvier 1984 dans le journal Le Devoir, le conseil scolaire de l'île de Montréal, entre autre Madame Laperrière, soulignait justement les difficultés d'intégration des élèves immigrants aux écoles françaises. Ces difficultés sont de deux ordres: a) linguistique b) socioculturel, et dues au fait que ces enfants sont "victimes de la pauvreté, du racisme et de l'ignorance culturelle de leurs camarades et de la non-maîtrise de la langue française." Etant donné que ces élèves confrontent des problèmes, par contre, ils ne produisent pas ou ne réussissent pas adéquatement à l'école. Ils sont tout de suite confondus aux enfants qui ont des difficultés d'apprentissage et d'adaptation scolaire et parqués dans les classes spéciales destinées à ces derniers.

Parmi ces élèves se retrouvent beaucoup d'Haïtiens. En effet, il existe en Haïti une dualité linguistique: français-créole. Cela s'explique d'ailleurs. Depuis 1804, le français est la langue officielle du pays, la langue de l'administration, la langue d'une élite; une "langue de valeur" parlée et comprise par dix à quinze pour cent de la population seulement, tandis que le créole est la langue du peuple et de la masse (langue nationale si l'on veut); la langue des faibles et des opprimés. Ainsi donc, on comprendra aisément pourquoi la plupart de ces élèves issus en grande partie de la classe défavorisée et du prolétariat et provenant de milieux ruraux où les écoles fonctionnent à rabais (point de vue qualitatif) connaissent des difficultés de cet ordre (linguistique).

Nous devons rappeler qu'à partir de 1970, l'immigration canadienne, se faisant plus humaine sous l'angle de la réunion des familles et du parrainage, a facilité l'entrée au pays de milliers de ruraux. Donc, une fois arrivés au Québec, ces élèves sont classés dans les écoles en fonction de leur âge et non par rapport à leur niveau académique et se trouvent quelquefois à un 
niveau (degré) supérieur qui ne correspond pas à leur véritable "background scolaire." Il s'ensuit de toute évidence ce que l'on sait: l'échec que l'on attribue à une mésadaptation de l'élève au milieu scolaire. Disons tout de suite que les élèves haïtiens nés au Québec ne souffrent pas de ce genre de problème.

D'autres causes peuvent expliquer l'inadaptation de la plupart des élèves haïtiens au milieu scolaire québécois. Nous avons déjà évoqué le mauvais fonctionnement des écoles en Haïti et particulièrement les écoles rurales. Des professeurs non qualifiés n'ayant pas de formation pédagogique requise y enseignent. Les enfants sont par conséquent mal préparés et ne sont pas prêts à affronter les réalités des écoles québécoises. Les programmes et les méthodes d'enseignement diffèrent énormément. Les relations ou les interactions professeurs/élèves sont tellement ouvertes et "décontractées" aux yeux de l'immigrant, qu'elles portent à penser à une "permissivité excessive" à l'opposé de l'école haïtienne qui prône encore l'autorité du professeur entretenant des relations distantes entre lui et ses élèves. Tout cela ne contribue-t-il pas à désorienter l'élève dans un premier temps?

De plus, les parents (la plupart d'entre eux) ne sont pas suffisamment instruits pour pouvoir aider l'élève à se rattraper et à remonter la pente. Les difficultés, au lieu de s'atténuer, s'intensifient au contraire. Au niveau des relations socio-familiales, des problèmes surgissent également. Le plus souvent, le père et la mère travaillent; frustrés, également désenchantés par ce qu'ils vivent dans leur milieu de travail. Les enfants eux aussi défoulent à la maison ce qu'ils vivent dans leur milieu scolaire, victimes eux aussi de leur différence raciale et culturelle. Les interactions familiales s'aggravent. Les relations parents/enfants deviennent tendues. Autant de facteurs contribuant à rendre difficile l'adaptation ou l'intégration de l'élève.

Il serait faux de prétendre que les difficultés d'adaptation des élèves haïtiens au milieu scolaire québécois proviennent seulement du fait qu'ils maîtrisent mal la langue française. En somme, ceci ne constitue que la pointe de l'iceberg. Nous avons essayé de dégager quelques-unes des raisons. Il y en a encore. Le conseil scolaire de l'île de Montréal dans une optique tout à fait semblable à la nôtre s'oriente dans cette voie et la plupart des recherches faites dans ce domaine "portent à croire que le climat socio-culturel inspiré par le programme ne s'oriente pas suffisamment dans une perspective interculturelle et ne permet pas une intégration sociale aussi rapide que pourrait de laisser entendre l'instrumentation linguistique des immigrants" (Kerlegrand, recherche sur les classes d'accueil, mémoire de maîtrise à l'Université de Montreal). Cette réflexion est aussi vraie dans le cas des élèves haïtiens que pour les autres groupes ethniques. 
Jean Kerlegrand, professeur de français langue seconde, est détenteur d'un baccalauréat spécialisé en anthropologie et diplômé en éducation de l'Université McGill. Il possède également une maîtrise en mesure et évaluation en éducation et poursuit actuellement des études doctorales en administration scolaire à l'Université de Montréal. 